\title{
Reflexiones sobre inclusión y educación superior
}

Sandra Acevedo Zapata ${ }^{1}$

\begin{abstract}
Resumen
Este artículo $^{2}$ presenta el trabajo descriptivo realizado en torno a la categoría de inclusión y las propuestas que hoy pueden afectar a las instituciones de educación superior. Para ello se han retomado las propuestas políticas colombianas lideradas por el Ministerio de Educación Nacional para garantizar el acceso a las poblaciones vulnerables. Se analiza el papel que deben cumplir los sistemas que integran la sociedad y en las conclusiones se hacen algunas recomendaciones que se pueden tener en cuenta en las instituciones de educación superior que buscan integrar estas propuestas de inclusión del gobierno y que pueden tener un papel fundamental en su implementación, además de esto se ha enriquecido la reflexión con los análisis de algunos expertos en prospectiva y su mirada sobre aspectos que también aportan a los procesos educativos en américa latina en los próximos años.
\end{abstract}

Palabras clave: inclusión, calidad, educación superior, diversidad y población vulnerable. 


\title{
Reflections on inclusion and higher education.
}

\begin{abstract}
This study presents a descriptive work done around the category of inclusion and the proposals that today can affect to the higher Education institutions. For this Colombians political proposals led by the Ministry of National Education have been resumed to guarantee access to the vulnerable populations. The role that has to fulfill the systems that integrate the society is analyzed. Furthermore, in the conclusions are included recommendations that can be taking in account for higher Education institutions that look to integrate these government proposals of inclusion. These could have a fundamental role in its implementation. In addition to this, the discussion has been enriched with the analyses of some experts in prospective and their opinion on aspects that also contribute to the educational processes in America Latin in the next years.
\end{abstract}

Key words: inclusion, quality, higher education, diversity and vulnerable population.

\section{Reflexiones sobre la categoría de inclusión y sus orígenes}

Presentar la noción de inclusión implica mostrar cómo se dio el proceso a través del tiempo. Inicialmente la escuela se pensó exclusivamente para las personas «normales» y los otros, quienes tenían alguna dificultad o presentaban discapacidades se recluían en su casa o en escuelas dedicadas a la educación especial para ser rehabilitados o habilitados, y de esta manera cumplir algunas actividades en la vida social. Sin embargo, se propuso una impronta de integración al aula regular en el foro mundial de Jomtiem (1990) «Educación para todos» y en el encuentro en Salamanca (1994) se les nominó como sujetos con necesidades educativas especiales. Luego se dio un proceso donde la intención de integración se fue trasformando en atención a la diversidad, y hoy en Colombia se está retomando la noción de inclusión a partir de las políticas programas estatales que buscan lograr la equidad en los procesos educativos. 
La búsqueda de la atención a la diversidad hace que entren en esta mirada muchas formas de ser diferente; están quienes presentan diferencias cognitivas, sensoriales, físicas, etarias y por condiciones sociales o culturales de quienes se consideran excluidos por el sistema educativo homogenizante.

La propuesta de inclusión busca que las personas puedan recibir una educación que les permita potenciar al máximo el desarrollo social y académico a partir de sus posibilidades en grupos de estudiantes sin las etiquetas propias de la exclusión.

Teniendo en cuenta uno de los principios del liberalismo económico, la participación en el mercado desde la diversidad de ofertas, lo cual ha movilizado la inserción de poblaciones como las mujeres al mercado laboral y ahora se hace también pertinente en este panorama, la necesidad de que todas las poblaciones diversas participen en las dinámicas del mercado. Este es un avance muy importante, como oportunidad para que la sociedad sea capaz de reconocer y asumir que las minorías son una gran mayoría. En este sentido Gentili y Frigotto, (2000: 21), nos plantea que: «La normalización de la exclusión comienza a producirse cuando descubrimos que, al final de cuentas, en nuestras sociedades hay más excluidos que incluidos».

Por tanto, la diversidad funcional que quiso sustituir a términos como discapacidad, propone a las personas en esta situación como seres valiosos por su diversidad. Esta perspectiva se asumió en el «Foro de Vida Independiente», realizado en España en el año 2005, donde se buscó superar la comprensión del cuerpo humano, desde la modernidad, cuando se concebía como una máquina, de la cual se pretendía conseguir la docilidad política y la utilidad económica de las personas, en correspondencia con los descrito por Foucault (2001). Este foro permitió configurar una nueva forma de enunciar y reconocer que las personas poseen unas características específicas, las cuales les permiten adaptarse y responder con exigencias del mercado y lograr diversos niveles de autonomía y resignificación de sus posibilidades sociales, laborales y académicas.

Es importante retomar el modelo de la diversidad, en el cual se asume la diferencia como un atributo inmanente al ser humano y a su existencia, mientras que la normalidad es una creación estadística puramente instrumental que no reconoce lo inconmensurable del sujeto. Los principales elementos que aporta el modelo de la diversidad es el cambio de paradigma, superando el imaginario del cuerpo perfecto y normal en la perspectiva biomédica, respetando y reivindicando la dignidad humana y esencialmente la autonomía moral. 
Asimismo en el artículo 2 de la «Declaración universal sobre el genoma humano y derechos humanos», se explicita que «Cada individuo tiene derecho al respeto de su dignidad y derechos, cualesquiera que sean sus características genéticas. a) Esta dignidad impone que no se reduzca a los individuos a sus características genéticas y que se respete el carácter único de cada uno y su diversidad» (Unesco,1997:140)

\section{La inclusión en el sistema educativo}

En el proceso descrito se ha impuesto la necesidad de implementar programas de gobierno que realicen la inclusión educativa, la cual requiere de un abordaje con nuevos significados del mundo y del Otro. Desde una perspectiva que permita comprender qué sucede al integrar en la escuela y especialmente en la educación superior, qué implica garantizar el ingreso y la permanencia de sujetos diversos en el sistema educativo, ahora con el apoyo de ambientes virtuales que deben facilitar este proceso.

Una responsabilidad fundamental es la construcción y afirmación de la identidad, no de una única, Sklyar (2002) nos propone esta discusión en su artículo Alteridades y pedagogías ¿Y si el Otro no estuviera ahí?, en el que nos invita a reflexionar sobre la construcción de la propia imagen, la cual se da a partir de la relación que establezco con el otro como sujeto que reconozco y del lugar que le doy; por tanto, cada uno significa su propio «Yo» fruto de la interacción que posibilita develar esa imagen por el «Otro».

Sklyar nos recuerda que en la escuela se vivencia en la cotidianidad un paradigma de incompletitud que afecta la relación pedagógica: a) la incompletitud según su condición diferencial cultural o física, b) la incompletitud reconocida como negativa y c) la impronta de la educación de complementar los que le falta al Otro. El riesgo que existe es que se irrespete al Otro por cómo es mirado y condicionándolo en lo que puede llegar a ser, por tanto es necesario reflexionar sobre cómo trasformar nuestra mirada de integradora a una verdadera mirada para la inclusión.

\section{Pasar de integración a inclusión}

La necesidad de pasar de la integración a la inclusión implica generar cambios retomando la filosofía inclusiva, que no restringe a la identificación y caracterización estadística de los estudiantes incluidos; por tanto se requieren cambios de orden psicopedagógico que permitan y potencien el despliegue subjetivo de los sujetos de todas las poblaciones diversas, con apuestas 
institucionales que incidan en lo curricular y pedagógico, que se reflejen en la manera en que se enseña, cómo se aprende, cómo se evalúa y principalmente cómo se da la relación pedagógica. Esto con el ánimo de brindar estas posibilidades de despliegue en múltiples sentidos, con la medición de la flexibilidad curricular y un verdadero seguimiento y acompañamiento a los estudiantes.

\section{La inclusión en la política colombiana}

El Plan Nacional de Desarrollo 2010 - 2014 promueve la protección social, la educación, el acceso, la permanencia, pertinencia y la calidad. Esto se evidencia en la búsqueda que igualdad que se propone en el plan con el desarrollo de las siguientes estrategias:

\section{BUSCANDO LA IGUALDAD DE OPORTUNIDADES PARA TODOS LOS COLOMBIANOS}

$>$ Primera infancia y niñez (atención integral)

$>$ Formación de capital humano (calidad y pertinencia)

$>$ Salud (unificación de planes y regulación de servicios excl.)

Juntos contra la pobreza extrema (acompañamiento)

$>$ Generación de ingresos (sistemas de intermediación laboral)

$>$ Población desplazada (goce de derechos y cesación)

$>$ Inclusión social (grupos étnicos, género)

Cultura (Industrias culturales, Programa de lectura, Patrimonio)
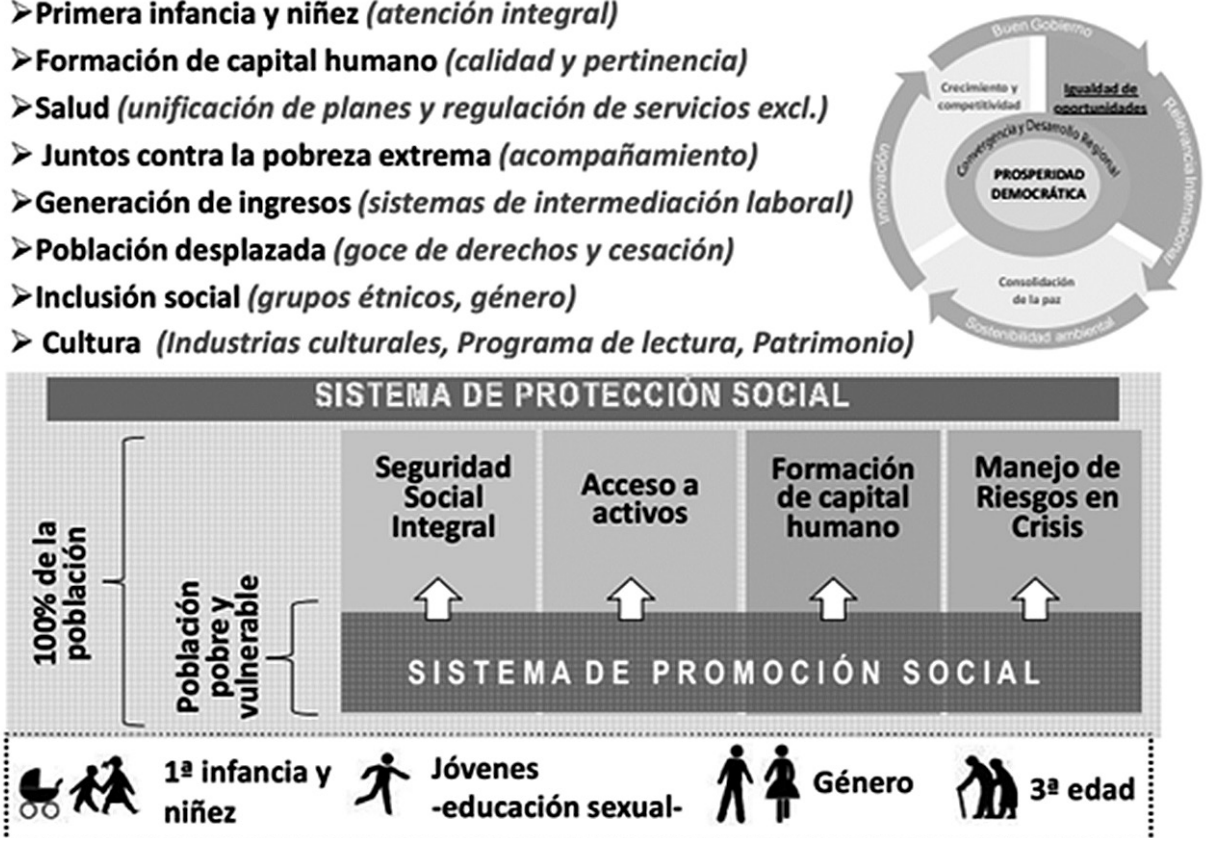

Figura 1. Plan Nacional de Desarrollo (2010 -2014).

La inclusión es un propósito fundamental que busca estrategias de igualdad y de oportunidades para toda la población: ejemplo de ello es la igualdad de género, en cuanto a la reducción de la violencia intrafamiliar, generación de condiciones equitativas de remuneración salarial, el reconocimiento de su aporte a la economía del cuidado y la responsabilidad frente a la crianza de los hijos. 
Se propone el desarrollo de estrategias y políticas diferenciadas que atiendan las diversas poblaciones, generando igualdad de oportunidades a grupos étnicos (población palenquera, afrocolombiana y raizal, pueblo rom-gitano y población indígena), población en situación de discapacidad, los jóvenes y los adultos mayores y población en situación de discapacidad.

La inclusión se presenta en las políticas como la igualdad de oportunidades y el desarrollo social integral para mejorar la calidad de vida de las poblaciones vulnerables y con necesidades especiales. Vale la pena resaltar que se concibe la calidad de vida como la posibilidad de acceso e inserción laboral, estabilidad económica, salud, el acceso a educación que propicie igualdad de oportunidades y acceso a actividades deportivas, artísticas y de recreación.

En la educación superior a distancia y virtual, se organiza y desarrolla como un grupo de estrategias metodológicas con el apoyo de las Tecnologías de la Información y Comunicación (TIC), que pretenden ampliar posibilidades de acceso a la educación, generando vías y modalidades alternativas para el ingreso a un mayor número de estudiantes, logrando una mayor cobertura y facilidad de acceso a todas las poblaciones antes marginadas por su ubicación territorial o por sus condiciones diferenciales.

La educación a distancia propende por un sistema educativo abierto que busca garantizar la formación integral de los sujetos a través de la autogestión del aprendizaje con el uso de diversos medios y mediaciones pedagógicas. En este sistema es fundamental retomar la experiencia de los diferentes actores del proceso educativo, tanto de sus necesidades como de sus potencialidades para mejorar las condiciones de vida de los estudiantes y sus comunidades.

\section{Metodología}

El objetivo general de esta investigación fue identificar y analizar la posibilidades de la inclusión en la educación superior en Colombia; y como objetivos específicos identificar las categorías implícitas en la apuesta por la inclusión en las políticas y mecanismos implementados en la educación superior en Colombia, sistematizar y analizar dichas políticas, y por último, divulgar los resultados de la investigación a la comunidad académica nacional e internacional. La metodología utilizada se enmarca en la perspectiva cualitativa y es una investigación descriptiva y de análisis documental y contempla las siguientes etapas:

Etapa exploratoria. Configuración del problema y rastreo documental de políticas y proyectos.

Etapa Descriptiva. Identificación de categorías y aplicación de instrumentos de recolección de información. 
Etapa de sistematización. Sistematización de información arrojada por los instrumentos a través de matrices de doble entrada entre categorías y actores, documentos y fuentes, identificando las principales posibilidades entre las apuestas en las políticas en educación superior por la inclusión con el uso de mediación virtual.

Etapa analítica. Análisis de posibilidades entre la inclusión y la calidad en la educación superior con el uso de la mediación virtual, el caso de la universidad colombiana.

Etapa proyectiva. Realización del informe y divulgación a través de una ponencia en un evento y construcción de un video y un artículo para presentar en revista indexada.

\section{Recomendaciones desde la prospectiva para la inclusión en educación superior}

Retomando lo encontrado en el proceso investigativo, es necesario pensar en una visión prospectiva, lo cual implica corregir rutas y construir escenarios a futuro, para comprometernos como sociedad con estos propósitos. En ese sentido, es fundamental reconocer el alto riesgo que se presenta en América Latina en lo referente a una explosión poblacional y tecnológica, la cual según Machado (2011) tiende a aumentar la inequidad y bajar la calidad de vida. Para lograr superar esta situación es necesario replantear la educación. Educar para la disminución de las desigualdades con nuevas propuestas para tratar las poblaciones o personas con condiciones económicas, culturales, sociales, físicas y cognitivas con el uso de las tecnologías de la información y la comunicación.

La importancia en la sociedad del conocimiento implica la apuesta por mejorar la educación con el uso de las tecnologías de la comunicación para potenciar la investigación y la construcción de conocimiento, atendiendo a los nuevos desafíos que plantean la inserción, internacionalización y la globalización. Según Machado, las demandas en el ámbito de la educación para el 2020 serían:

“... a) asegurar el desarrollo humano y mejorar la calidad de vida de las personas, considerando la preservación del medio ambiente, la superación de la pobreza y el logro de la educación inclusiva; b) fortalecer el desarrollo de la ciencia y la tecnología; c) contribuir a la integración y al fortalecimiento de la ciudadanía ; d) mejorar las condicione del mundo del trabajo" (2011:6).

En 1990 en Jomtiem, Tailandia, en el marco de la Declaración Mundial sobre Educación para Todos, en el punto «Satisfacción de las necesidades básicas de aprendizaje», se hizo énfasis en el «aprendizaje básico (conocimientos teóricos 
y prácticos, valores y actitudes) necesarios para que los seres humanos puedan sobrevivir, desarrollar plenamente sus capacidades, vivir y trabajar con dignidad, participar plenamente en el desarrollo, mejorar la calidad de vida, tomar decisiones fundamentadas y continuar aprendiendo».

Se espera que en el 2020 se haya logrado satisfacer la necesidades de todos los miembros de la sociedad como sujetos iguales que hayan tenido la posibilidad de desarrollar su creatividad, la flexibilidad para adaptarse a diferentes situaciones y a los cambios, así como también hayan desarrollado su capacidad emprendedora, con integridad ética. Lo anterior en paralelo a sus responsabilidades como sujeto, en cuanto a enriquecer la historia cultural, espiritual y lingüística, promoviendo la educación de los demás, actuando de manera comprometida con el medio ambiente y la justicia social, y siendo tolerante con las creencias y representaciones políticas y sociales.

Se espera además que el sistema de educación superior haya logrado superar los problemas de financiación y logre apoyar, de manera diferenciada, a quienes más lo necesitan, así como generar procesos que equilibren las condiciones sociolingüísticas relacionadas con el capital cultural, el cual ha generado veladas formas de exclusión en el trascurso de la historia humana.

Miklos y Arrollo, quienes analizan cuatro escenarios utilizando el análisis estructural, y retomando uno de ellos, como real y posible, muestran que:

“...la globalización y las nuevas tecnologías se expanden a todo el planeta en el marco de las políticas regulatorias nacionales e internacionales, lo que reduce las brechas de la mundialización. La socialización democrática de las tecnologías facilita el acceso a la educación y la conectividad se masifica cubriendo a amplios sectores sociales, sin embargo, permanecen rezagos sociales y brechas digitales. La educación se ha articulado como un bien público internacional, que facilita responder a las necesidades de la población, permitiendo crecientes niveles de calidad y altas tasas de cobertura de la educación en América Latina en el marco de modelos flexibles y pertinentes apoyados en las TIC. Sin embargo, la reconfiguración de las instituciones marcha lentamente y los procesos de innovación en la práctica docente generan conflictos sindicales, gremiales y epistemológicos que "lentifican" el cambio.

Se han instrumentalizado múltiples procesos de reformas educativas que promueven la expansión de la calidad, tanto en las instituciones públicas como privadas que se enfrentan a los gremios conservadores de profesores y a otras zonas y agentes conservadores. Las instituciones públicas diferencian mecanismos de financiación a través de la vinculación con la industria privada y esquemas personalizados de becas, en un escenario donde la educación virtual se valora como u mecanismo idóneo para ampliar la cobertura y el acceso a la educación, y contribuir a cerrar las brechas de conocimiento" (2008:61). 
Para finalizar, y considerando lo anotado en las páginas anteriores, a continuación se sintetizan algunos elementos que son necesarios repensar y reconstruir a la hora de pensar en la inclusión como aspecto fundamental en el campo educativo y otros escenarios.

- Construir el escenario futurible descrito por Miklos y Arrollo, exige el trabajo conjunto de diferentes sectores de la economía, la sociedad y la educación, unidos por estos propósitos comunes de acercarse a mejorar la calidad y responder a las necesidades de los sujetos en su diversidad y especificidad.

- La inclusión educativa se constituye en una estrategia fundamental en el proceso de aseguramiento de la calidad de la educación, requiere de una ética del reconocimiento del "Otro", para cumplir con el propósito de lograr la equidad en todos los sujetos y de los grupos poblacionales a los cuales pertenecen.

- Es necesario un cambio en el sistema educativo que supere los prejuicios sobre la educación a distancia y que potencie el uso de las TIC con el e-learning y el b-learning, para llegar a los más necesitados y excluidos del sistema educativo. Lo anterior requiere un alto nivel de compromiso que se evidencia, entre otras cosas, en garantizar la interacción entre el docente y el estudiante.

- El desarrollo del proyectos políticos de inclusión se materializa en los programas de prevención de la deserción e implica inversión y reformas psicopedagógicas en los procesos de seguimiento y acompañamiento a los estudiantes y sus problemáticas tanto en la educación presencial como en la educación a distancia y virtual.

- Es necesario retomar los aportes de todos los actores que participan en los procesos de formación que potencian sus posibilidades, sistematizar las experiencias y aprender de las voces de quienes protagonizan procesos de inclusión.

- Las estrategias institucionales para generar procesos inclusivos requieren ajustes en la política con modelos realmente inclusivos, que trasformen tanto los procesos administrativos, como la formación de todos los actores que interactúan con las diversas poblaciones que llegan a las instituciones de educación superior.

- La educación inclusiva pasa por adecuaciones tecnológicas, pero más allá de este aspecto, es fundamental una trasformación epistemológica y ética de todos y cada uno de los actores de la comunidad educativa. Asumir los avances tecnológicos que permiten mayor accesibilidad, se requiere la evaluación y 
revisión de los proyectos y de los programas que se han desarrollado. No hay que olvidar que en la instituciones de educación superior (IES) es donde se han apropiado aplicativos, softwares y medios educativos exitosos con las poblaciones diversas de los estudiantes.

- Se requiere la construcción del estado del arte de programas de experiencias y de investigaciones, así como también conocer los proyectos de educación inclusiva desarrollados por la UNAD teniendo en cuenta su esencia misional en torno a la inclusión. Lo anterior debe ir acompañado de la revisión de las experiencias a nivel a nacional e internacional.

- También es necesaria la caracterización de las diversas poblaciones que se forman y trabajan en las IES, recogiendo sus testimonios, necesidades y experiencias en su proceso de educación virtual, con el objetivo de identificar las posibles barreras institucionales, y con ello permita generar nuevas alternativas y ajustes para resolver las situaciones que se presentan.

- Las IES requieren investigar acerca de la pertinencia del currículo de las licenciaturas y posgrados de formación de docentes. También es importante la revisión y análisis de las estrategias pedagógicas y actividades académicas desarrolladas por los docentes en relación con las diversas poblaciones.

- Las IES necesitan diseñar una política que promueva una participación activa y decidida de toda la comunidad académica, especialmente de los propios estudiantes; una estructura que reconozca la importancia y particularidades del enfoque de educación inclusiva, aplicando sus procesos de autoevaluación de los programas de acompañamiento y seguimiento a las diversas poblaciones y de los programas de bienestar institucional, que incluya a estudiantes y egresados.

- Para el caso específico de la UNAD, se requiere revisión y establecimiento de políticas institucionales, que consideren los protocolos y convenciones internacionales, con el ánimo de adecuarlos a nuestras condiciones y regulaciones, lo cual requiere la identificación de las necesidades específicas de las poblaciones de cada región donde tiene presencia la institución.

- Construir colectivamente en foros, seminarios y congresos, con las diversas poblaciones que integran las IES, los lineamientos, políticas y procesos tanto académicos como administrativos de acuerdo con la educación inclusiva y permitir, desde estas redes, la evaluación permanente sobre la implementación de estos procesos.

- Diseñar e implementar procesos de reestructuración de los programas de formación de docentes, articulados a los procesos de acreditación, reforma curricular, innovación tecnológica y administrativa para incorporar la educación inclusiva a la formación de docentes con perfil inclusivo. 
- La formación de docentes implica la construcción del perfil de los docentes, quienes deben ser formados en competencias para construir y trasformar currículos, con innovación de contenidos, estrategias pedagógicas y evaluativas. Además, se requiere de docentes que sepan investigar sobre su propia práctica pedagógica en lo relacionado con la formación de estudiantes de diversas poblaciones y tengan la oportunidad de desarrollar prácticas pedagógicas inclusivas como parte fundamental de su formación.

\section{Referencias bibliográficas}

Foro de Vida Independiente. 2005. Apuntes sobre el documento: líneas básicas para un modelo de atención a las personas dependientes. Disponible en: http://www. minusval2000.com/relaciones/vidalndependiente/apuntes_sobre_documento_ccoo. html

Foucault, Michel. 2001[1974-1975]. Los anormales. Curso en el Collège de France (19741975). México: FCE.

Gentili, Pablo y Frigotto, Gaudencio. 2000. La ciudadanía negada. Políticas de exclusión en la educación y el trabajo. Buenos Aires: Clacso.

Machado, Ana Luiza. 2011. La educación en América Latina y el Caribe: Visión prospectiva al año 2020. Consultado en: http://www.reggen.org.br/midia/documentos/ laeducacionenamericalatina.pdf

Milklos, Tomás y Arrollo, Margarita. 2008. Una visión prospectiva de la educación a distancia en América Latina. Universidades. Vol. LVIII (37): 49-67.

Sklyar, Carlos. 2002. Alteridades y pedagogías ¿Y si el Otro no estuviera ahí?, Educação \& Sociedade, año XXIII, 79: 85-123.

Unesco. 1997. Declaración universal sobre el genoma humano y los derechos humanos. Proclamada por la Conferencia General de las Naciones Unidas para la Educación, la Ciencia y la Cultura en su 29 reunión. Adoptada por la Asamblea General en su Resolución 53/152, del 9 de diciembre de 1998.

Unesco. 1990. Declaración mundial sobre educación para todos y marco de acción para satisfacer las necesidades básicas de aprendizaje. Jomtien: Unesco. 